\title{
Aubert Gauthier, Les Révoltes du papier timbré, 1675.
} Essai d'histoire événementielle

\section{Guy Saupin}

\section{Q OpenEdition}

1 Journals

Édition électronique

URL : http://journals.openedition.org/abpo/2995

DOI : $10.4000 / a b p o .2995$

ISBN : 978-2-7535-4130-6

ISSN : $2108-6443$

\section{Éditeur}

Presses universitaires de Rennes

\section{Édition imprimée}

Date de publication : 15 avril 2015

Pagination : 199-202

ISBN : 978-2-7535-4128-3

ISSN : 0399-0826

\section{Référence électronique}

Guy Saupin, «Aubert Gauthier, Les Révoltes du papier timbré, 1675. Essai d'histoire événementielle», Annales de Bretagne et des Pays de l'Ouest [En ligne], 122-1 | 2015, mis en ligne le 15 avril 2015, consulté le 23 septembre 2020. URL : http://journals.openedition.org/abpo/2995 ; DOI : https://doi.org/ $10.4000 /$ abpo. 2995 
AuberT, Gauthier, Les Révoltes du papier timbré, 1675. Essai d'histoire événementielle, Rennes, PUR, coll. « Histoire », 2014, 717 p.

L'ouvrage que Gauthier Aubert a tiré du mémoire original de son HDR est triplement provocateur. Pourquoi revenir sur une crise très connue qui a suscité une gamme déjà très diversifiée d'interprétations, en défendant une approche événementielle alors que ce genre passait il y encore peu de temps pour la manière la plus éculée de faire de l'histoire, et en plaçant sa démarche dans le registre de l'essai, terme sous-entendant habituellement un texte court centré sur la défense de quelques grandes idées, alors qu'il s'agit d'une étude très détaillée de plus de 700 pages ? L’analyse des révoltes bordelaises et bretonnes de 1675 se déroule en 13 chapitres, regroupés en quatre parties selon un phasage chronologique, solidement encadrés par une introduction et une conclusion, tout en intégrant un prologue très intéressant centré sur les emboîtements d'échelle jusqu'à la guerre de Hollande et un épilogue s’attaquant à la délicate interprétation de la sortie de crise.

Le cœur de l'affaire est bien sûr dans l'argumentation déployée pour justifier cette mise en avant de l'événement, au service d'une histoire « concrète ». Plusieurs positionnements méritent qu'on s'y arrête. L'auteur note avec lucidité qu'en Bretagne il n'existe guère de frontière étanche entre production historique et enjeux mémoriels et identitaires comme l'actualité contestataire vient de nous le rappeler. Retrouver l'événement s'affiche donc comme un manifeste pour clarifier les relations entre mémoire et histoire en passant de l'une à l'autre. Par ailleurs, il souligne le risque d'entrer dans l'interprétation d'une crise en mettant cette dernière au service d'un autre objet historique tel que la nature de la société d’Ancien Régime ou de l'absolutisme dans le cas qui nous intéresse, avec tous les biais téléologiques que ce cela peut induire. Les leçons de l'interdisciplinarité, et principalement la fréquentation des sociologues et des politistes, soulignent que « la quête du comment est plus féconde que celle du pourquoi » puisqu'il ne saurait exister de déterminisme à la mobilisation. Partant d'une pluralité de causes possibles, le plus intéressant est de comprendre comment on bascule de la contestation à la révolte, comment des actions programmées ne démarrent pas ou comment un brasier s'enflamme sur fond de récriminations. Les questions du déclenchement des émeutes, de la contagion du mouvement insurrectionnel, des interactions entre tous les types d'acteur, de l'apparition de divers types dans le déroulement depuis l'entrée jusqu'à la sortie de crise sont ainsi au cœur de l'ouvrage et justifient la référence à une histoire " concrète » ou encore « matérielle ».

Gauthier Aubert s'intéresse aux conditions de rencontre entre un contexte et des opportunités, au croisement de la culture de la contestation violente avec celle de l'autorité démonstrative, mais aussi à l'intervention des forces de médiation dans une temporalité incertaine. Il alerte sur une tendance fréquente des historiens à adopter trop aisément le point de vue de l'État se présentant comme modernisateur et seul capable d'incarner l'intérêt général face à des acteurs locaux qui ne seraient porteurs que de leurs intérêts individuels et catégoriels. Ce manque de distanciation peut conduire à une incompréhension des pratiques politiques, comme par exemple à traduire en termes de complicité l'attentisme des notables dans la phase première de la révolte et en termes de lâche retournement leur ralliement à l'ordre royal dans la phase de la répression, alors qu'il s'agit plutôt d'un mode traditionnel de rétablissement de la paix dans la préservation de l'ordre communautaire dans l'idéal classique du bon gouvernement.

Au centre de la démarche se trouve une interrogation sur la prise de décision politique, dans une situation où le poids de l'international à travers la guerre rencontre les questions de vie quotidienne de sujets qui doivent s'acculturer au renforcement de la pression fiscale et dans une chaîne relationnelle où interviennent des acteurs sociaux très variés, avec une mention spéciale pour le monde médian des petits robins, dans l'articulation entre les élites et le peuple. Les pages montrant le processus de mobilisation et les divers types de réaction possibles au sein de la milice bourgeoise, autre cadre essentiel où s'expriment les convictions de l'élite populaire, sont vraiment passionnantes. Tout le renouveau historiographique sur cette structure fondamentale de l'organisation sociale et politique des communautés urbaines est ici mobilisé pour donner des clés de lecture très éclairantes. L'ouvrage défend l'idée du caractère graduel d'une réponse que le pouvoir royal essaie de proportionner aux enjeux perçus. On y observe un mélange d'intimidation et de négociation, deux faces d'une même méthode avant tout pragmatique. Le fait que Louis XIV ait pu réellement s'inquiéter de la conjonction des révoltes intérieures et du front extérieur de la guerre de Hollande n'a guère été retenu jusqu'à présent. Or il fallait continuer à financer une 
guerre qui n’avait plus rien de l'expédition aisée imaginée au départ tout en empêchant une extension géographique de la rébellion à l'intérieur du royaume. Temporisation et durcissement alternent selon les réactions des uns aux messages envoyés par les autres. Il n’existe pas de politique répressive dure systématique définie dès le départ, même si l'intransigeance sur le paiement ne faiblit pas. Tout ceci amène Gauthier Aubert à discuter pour le nuancer le schéma interprétatif d'Yves-Marie Bercé sur un avant et un après 1661 opposant une certaine mansuétude empreinte d'hésitations et une efficacité partielle de l'émeute à une défaite sans appel par l'application d'une méthode répressive « exacte et terroriste ». Là encore, le contexte de guerre internationale a sans doute pesé lourd.

L'ouvrage prend soin de s'interroger sur les grandes interprétations déjà proposées pour rendre compte des révoltes, tout en questionnant la dualité entre les émeutes rennaises successives et le soulèvement rural de Basse Bretagne. Si la fin d'un « âge d'or » peut concerner la Cornouaille intérieure, ceci aurait dû mobiliser beaucoup plus largement ; de plus, c'est assez inopérant pour Nantes et Rennes. La dureté sociale des règles du domaine congéable expliquent en partie le caractère anti-seigneurial de la révolte des Bonnets Rouges, originalité relevée depuis longtemps au sein de l'ensemble français renvoyant vers les mobilisations frumentaires et surtout anti-fiscales. Une réaction culturelle défensive contre une Réforme catholique jugée oppressante peut singulariser les bas Bretons ; reste à comprendre pourquoi d'autres zones géographiques présentant les mêmes caractéristiques n’ont pas bougé, sans compter l'impact sur la gestion de sortie de crise pour éviter l'affrontement suicidaire. Il est aussi une autre fracture culturelle qui pourrait jouer le rôle de boute-feu. Le rapport à l'écrit est certainement clivant, avec ceci de particulier que pour le menu peuple l'écrit se présente tout d'abord comme un acte officiel. Si le recours à l'acte n’est sûrement pas une nouveauté, il reste socialement très différencié. Son timbrage symbolise, avec la dure concrétisation du fisc, l'intrusion de l'État monarchique dans les affaires privées. Tout ceci s'inscrivant dans un sentiment de fragmentation de la communauté rurale avec un creusement des écarts socio-économiques et culturels.

Il est aisé de percevoir combien ce bilan historiographique laisse Gauthier Aubert insatisfait. Si cela peut nourrir un malaise, cela ne suffit pas à rendre compte du déclenchement, de l'extension et de la conclusion du mouvement émeutier dans sa double dimension sociale et géographique. Toujours en quête du comment, il s'oriente dans deux directions complémentaires qui concernent le rapport des sujets à l'État monarchique dans une implication directe avec la question du respect de l'ordre établi. Examinant d'abord la question d'une relative faiblesse de l'encadrement judiciaire des populations de Basse Bretagne à partir des actes du parlement, il pose l'intéressante question du sentiment d'une relative impunité favorable à la persistance du recours à la violence collective pour se faire entendre. Il en voit lui même les limites puisque les révoltes de Bordeaux, Nantes et surtout Rennes ont éclaté dans des villes surencadrées. Ajoutons que la limitation à l'échelon de la cour d'appel peut être quand même trop lointain pour des catégories sociales populaires plus habituées aux niveaux inférieurs de la justice royale. Mais comme il s'agit de perception, l'éloignement géographique pour les ruraux peut aider à la prise de risques. Reste à expliquer pourquoi l'évêché de Saint-Brieuc, qui présente les mêmes caractéristiques, n’a pas bougé.

La seconde interrogation renvoie au militaire dans une intéressante association de plusieurs dimensions. Même si la Bretagne est en train d'entrer comme frontière maritime stratégique dans la conception militaire du pouvoir royal avec l'installation du plus grand arsenal français à Brest, le soldat de l'armée de terre y reste un relatif inconnu. Par contre, les traditions de mobilisation défensive des populations du littoral contre les agressions ennemies sont encore très vivantes et même revitalisées. Pourrait donc s'additionner une habitude martiale aiguisée par la peur d'un ennemi qui ne vient pas (angoisse du débarquement hollandais en 1674), le sentiment d'éloignement de la force répressive intérieure et la peur du soldat inversement proportionnelle à sa méconnaissance réelle, car nourrie des informations sur les horreurs de ce « siècle de fer ». Cette approche a le grand mérite de reconstituer un arrière-plan politico-culturel à partir duquel les formes d'action peuvent prendre un caractère très varié selon l'évolution du contexte. Alors qu'une observation superficielle pourrait qualifier le mouvement protestataire d'erratique afin d'expliquer ses contradictions, une analyse plus fine conduit au contraire à soutenir la thèse d'une maturité politique avancée des populations en révolte qui ont appris de l'expérience du demi-siècle qui précède et qui savent alterner les actions et surtout sortir de l'engrenage fatal avant que celui-ci tourne au drame si le rapport de forces devient défavorable. D’où le 
très grand intérêt des réflexions sur la sortie de crise.

L’ouvrage rédigé par Gauthier Aubert est d'une très grande richesse. Extrêmement documenté, il suit de très près tous ses nombreux protagonistes dans leurs espaces géographiques, politiques et culturels. L'auteur s'interroge en permanence, soupèse tous les arguments, expose toutes les nuances et avance avec prudence ses préférences en termes d'interprétation. Toute inquiétude, un peu formelle quand on connaît l'auteur, d'avoir à avaler un indigeste pavé factuel sous prétexte de sacrifier à un renversement de perspective épistémologique, est vite dissipée. L'approche événementielle est bien au service d'une meilleure compréhension de l'histoire sociale du politique qui bénéficie ainsi d'un enrichissement certain. Est-on, comme l'annonce l'introduction, dans un abandon de l'utopie du pourquoi suite à la trop grande pluralité de causes possibles amenant immédiatement leurs contre-exemples, au profit d'une valorisation du comment qui permettrait de donner une meilleure lisibilité d'interprétation en aidant à résoudre les apparentes contradictions d'une analyse finaliste ? L'historien ne se débarrasse pas aussi facilement de la recherche des causes, même s'il s'agit de les exprimer en termes de visions interactives du problème par l'ensemble des acteurs concernés, le long de toute la chaîne relationnelle liant le roi à ses sujets. En refermant le livre, le lecteur a compris qu'il ne s'agit pas d'une alternative, de jouer l'un contre l'autre, mais bien d'associer le plus étroitement possible deux démarches complémentaires.

Grâce aux judicieux emboîtements d'échelle dans le temps et dans l'espace, la conclusion essentielle du point de vue de l'histoire sociale du politique est bien la progression de l'adhésion volontaire de la très grande majorité des élites sociales à l'ordre socio-politique contenu dans le modèle monarchique d'inspiration absolutiste incarné par Louis XIV. Les révoltes de 1675 illustrent la disparition des attitudes d'accommodement d'une violence contenue légitimée comme un appel à la négociation. Si le groupe médian rural ou urbain entretient encore une certaine ambiguïté, celle-ci n'est plus que de rumeurs et de papier sans presque jamais passer à l'acte. Le mimétisme socio-politique est bien à l'oeuvre. Ceci ne marginalise pas entièrement les catégories populaires qui sont amenées à approfondir leurs capacités d'appréciation stratégique et d'adaptation tactique dans leur autonomie d'action. En ce sens, plutôt que d'apparaître comme la fin du grand cycle rébellionnaire selon l'interprétation classique, les révoltes de 1675 peuvent donc être lues comme le début d'un cycle de transition menant à la judiciarisation des conflits du xvIII ${ }^{\mathrm{e}}$ siècle, exception faite des révoltes frumentaires.

Guy SAUPIN 